\title{
A Utilização da Difração de Neutrões na Determinação do Perfil de Tensões Residuais em Revestimentos por Soldadura
}

\author{
(The Use of Neutron Diffraction for the Determination of the in-Depth Residual Stresses Profile in Weld Coatings)
}

\author{
Maria José Marques ${ }^{1,2, *}$, A. C. Batista ${ }^{1}$, J. P. Nobre ${ }^{1}$, Altino Loureiro ${ }^{3}$, Joana R Kornmeier ${ }^{4}$ \\ ${ }^{1}$ CEMDRX, Departamento de Física, Universidade de Coimbra, Portugal \\ ${ }^{2}$ Departamento de Engenharia Física, Faculdade de Engenharia da Universidade do Porto, Portugal \\ ${ }^{3}$ CEMUC, Departamento de Engenharia Mecânica, Universidade de Coimbra, Portugal \\ ${ }^{4}$ FRM II, Technische Universität München, Garching, Alemanha \\ "mjvaz@fe.up.pt
}

\begin{abstract}
Resumo
A técnica de difração de neutrões é usada neste artigo para estudar amostras ferríticas, revestidas com aços inoxidáveis austeníticos através de soldadura por arco submerso. Este procedimento é frequentemente usado no fabrico de equipamentos de processo para as indústrias química e nuclear, por facilidade de execução e razões económicas. A principal desvantagem deste processo de revestimento é a fissuração que frequentemente ocorre na interface material base/soldadura, potenciada pela presença de tensões residuais resultantes da operação de soldadura, a qual pode ser minimizada com a realização de tratamentos térmicos de relaxação de tensões.

As amostras foram produzidas a partir de placas em aço ao carbono, tendo uma das superfícies sido revestida com dois tipos de aço inoxidável. Para a primeira camada foi usado um elétrodo EN 12072 - S23 122 L e para a segunda e a terceira camadas foi usado um elétrodo EN 12072 - S19 123 L. Após a soldadura, as amostras foram submetidas a um tratamento térmico de relaxação de tensões, durante 1 hora, à temperatura de $620^{\circ} \mathrm{C}$. Os perfis de tensões residuais obtidos por difração de neutrões evidenciam a relaxação de tensões residuais após o tratamento térmico realizado. A técnica de difração de neutrões revelou-se muito adequada na avaliação de tensões residuais neste tipo de ligações.
\end{abstract}

Palavras-Chave: Difração de neutrões; Tensões residuais; Revestimentos; Arco submerso.

Abstract: The neutron diffraction is a non-destructive technique, particularly suitable for the analysis of residual stress fields in welds. The technique is used in this article to study ferritic samples, coated by submerged arc welding using stainless steel filler metals. This procedure is often used for manufacturing process equipment for chemical and nuclear industries, for ease of implementation and economic reasons. The main disadvantage of that processes is the cracking phenomenon that often occurs at the interface between the base material and coatings, which can be minimized by performing post-weld stress relief heat treatments.

The samples analyzed in this study were made of carbon steel plates, coated by submerged arc welding two types of stainless steel filler metals. For the first layer was used one EN 12072 - S 2 U 2312 electrode, while for the second and third layers were used an EN 12 072 - $19123 \mathrm{~S}$ L electrode. After cladding, the samples were submitted to a post-weld heat treatment for 1 hour at $620^{\circ} \mathrm{C}$. The residual stress profiles obtained by neutron diffraction evidence the relaxation of residual stress given by the heat treatment.

Key-words: Neutron diffraction; Residual stresses; Cladding; Submerged arc welding.

\section{Introdução}

Os príncipios físicos da determinação de tensões residuais pelas técnicas de difração de raios X e por difração de neutrões são idênticos [1-3]. No entanto, enquanto a difração de raios $X$ analisa apenas uma pequena camada da ordem de alguns micróme-

Recebido em 22/01/2013. Texto final em 21/03/2013. tros ou dezenas de micrómetros, próxima da superfície do material, a difração de neutrões possibilita uma análise do material através da espessura da amostra, sem ser necessário proceder à remoção de camadas sucessivas de material, como acontece na técnica de difração de raios X. Recorde-se que a profundidade de penetração da radiação é função do comprimento de onda da radiação incidente, do coeficiente de absorção do material irradiado e das condições geométricas da montagem experimental. Assim sendo, e devido ao maior poder de penetração dos neutrões, a técnica de difração de neutrões torna-se particularmente indicada para a determinação da distribuição das tensões residuais em profundidade, em soldaduras e em amostras submetidas a 
tratamentos térmicos, entre outras aplicações.

\section{Determinação de Tensões Residuais por Difração de Neutrões}

Nas instalações nucleares de investigação denominadas habitualmente por "large-scale research facilities", existem instrumentos otimizados para a determinação de deformações e tensões residuais em materiais. Atualmente existem instalações deste tipo um pouco por todo o mundo, referindo-se em particular as instalações europeias, a saber:

- FRM II, Garching, Alemanha (www.frm2.tum.de), Figura 1 [4];

- HZB, Berlim, Alemanha (http://www.helmholtz-berlin.de) [5];

- PSI, Villigen, Suiça (http://www.psi.ch) [6];

- ISIS, Oxford, Reino Unido (http://www.isis.stfc.ac.uk) [7];

- LLB, Saclay, França (http://www-llb.cea.fr) [8];

- ILL, Grenoble, França (http://www.ill.eu) [9].

A Figura 1 ilustra uma vista da instalação onde foram realizadas as determinações de tensões residuais referidas neste artigo.

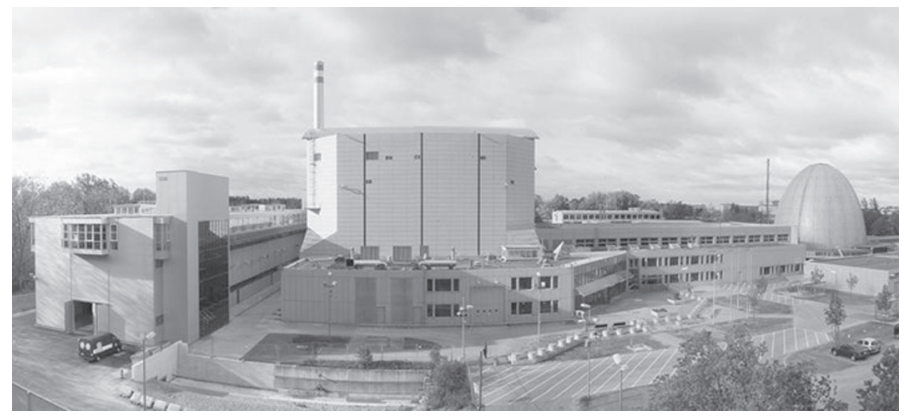

Figura 1. Reator nuclear de investigação FRM II da Universidade Técnica de Munique, Garching, Alemanha.

Os neutrões são obtidos a partir de núcleos atómicos ricos em neutrões, como o tunsgténio, urânio, tântalo ou chumbo [10]. Presentemente as fontes de neutrões produzem feixes com densidade de fluxo da ordem de $10^{15}$ neutrões $/ \mathrm{cm}^{2} \cdot \mathrm{s}$, existindo essencialmente dois meios de produção, a saber, as fontes contínuas e as fontes pulsadas, conforme se ilustra na Figura 2. Nas fontes contínuas os neutrões são produzidos por fissão do núcleo de urânio 235. No caso dos reatores nucleares, os elementos de combustão são grandes e contêm apenas $25 \%$ deste isótopo de urânio. No caso dos reatores de investigação os elementos de combustão são muito mais compactos e por isso são enriquecidos com este isótopo até $90 \%$ para se conseguirem obter grandes intensidades do feixe de neutrões. De realçar que a abundância natural deste isótopo é de apenas $0,7 \%$ e, para evitar simultaneamente a proliferação de armas nucleares, existe há alguns anos o encorajamento dos governos para que os reatores de investigação reconstruam os seus elementos de combustão para não necessitarem mais de $20 \%$ deste tipo de isótopo.

No caso das fontes de espalação, um feixe de protões lineares muito energético, (tipicamente $70 \mathrm{MeV}$ ), é produzido num acelerador linear e é injetado num anel de sincrotrão para atingir energias ainda mais elevadas $(500-800 \mathrm{MeV})$. Estes por sua vez são atirados contra um núcleo atómico com um elevado número de neutrões, como por exemplo o tungsténio 183 ou o urânio 238. A maior parte destas fontes opera em modo pulsado. A utilização de um ou outro tipo de reator para investigação depende do objetivo do estudo.

Comparativamente com outras técnicas de determinação de tensões residuais, a difração de neutrões tem a vantagem principal de ser um método não destrutivo de análise das tensões residuais em profundidade. Num material cristalino, os átomos dispõem-se num arranjo regular nas três direções do espaço. Nestes materiais a distância interplanar entre os planos de átomos está definida e é característica de cada material, se considerarmos o material sem tensões. Quando se irradia um sólido cristalino com um feixe de radiação monocromática, com comprimento de onda da ordem de grandeza da separação entre os átomos na estrutura cristalina, observa-se a existência de um feixe emergente (diz-se difratado) apenas para certos valores do ângulo entre o

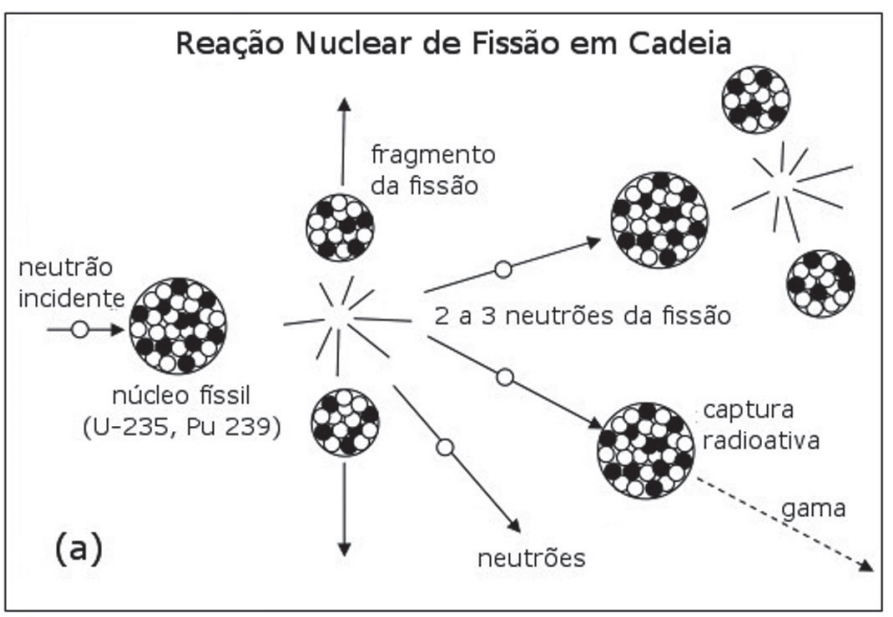

\section{Reação Nuclear de Espalação}

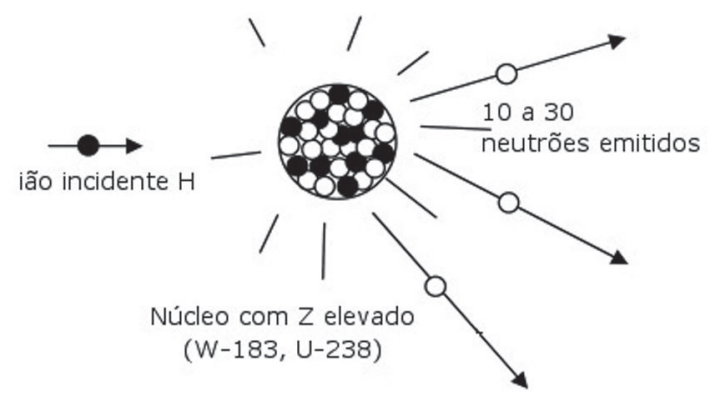

(b)

Figura 2. Tipos de fontes de neutrões: (a) fonte contínua: fissão do núcleo de U-235 e (b) fonte pulsada: reação de espalação. 


\section{Material sem tensões}

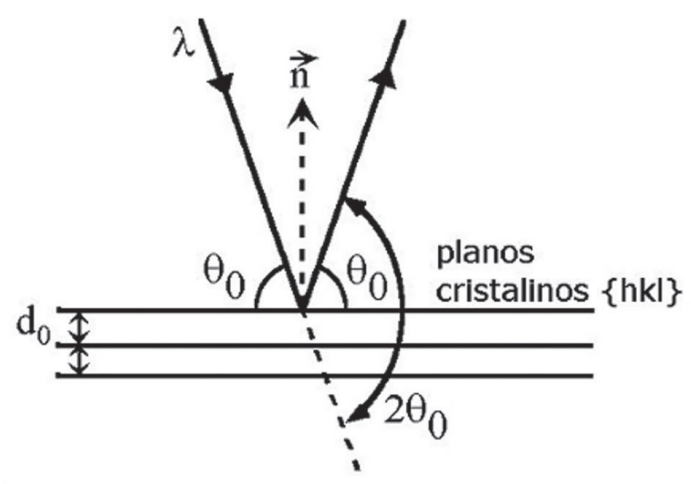

Material sujeito a tensões

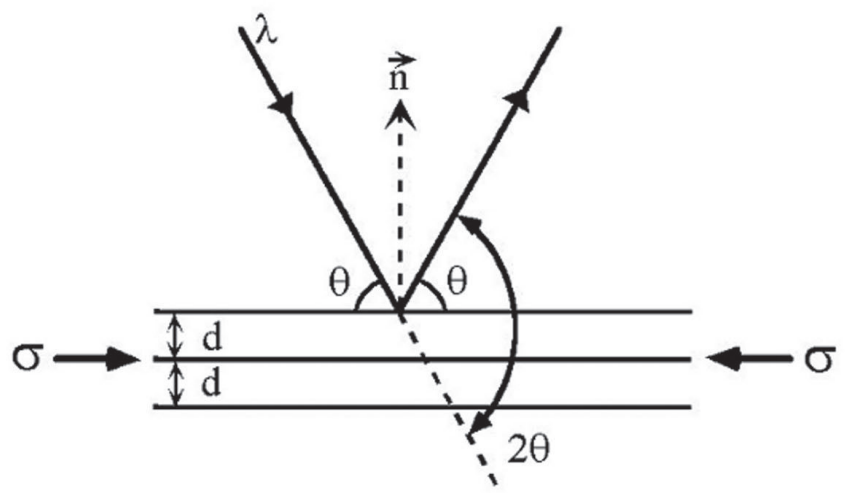

Figura 3. Planos cristalinos em condição de difração.

feixe incidente e o feixe emergente, o que se traduz pela lei de Bragg, explicitada pela equação 1,

$2 \mathrm{~d}_{\mathrm{hkl}} \sin \theta=\mathrm{n} \lambda$

onde $\lambda$ é o comprimento de onda da radiação, $d_{h k l}$ a distância interplanar da familia de planos cristalinos e $\theta$ o ângulo de difração (Figura 3).

Se o material for constituido por grãos orientados aleatoriamente, haverá sempre um conjunto de grãos em condições de difratar, qualquer que seja a orientação da amostra relativamente ao feixe incidente.

Num material policristalino, com grãos de pequena dimensão e livre de tensões, o espaçamento entre planos cristalinos, $\mathrm{d}_{0}$, não varia com a orientação dos grãos. Se a amostra estiver sujeita a uma tensão de tração, a distância interplanar passa a depender da orientação dos planos em relação à tensão aplicada. $\mathrm{O}$ espaçamento interplanar dos planos perpendiculares à direção da tensão aplicada aumentará, enquanto que a dos planos paralelos à tensão aplicada diminuirá, como resultado do efeito de Poisson. A deformação elástica num material pode assim ser determinada através da variação da distância interplanar, determinada experimentalmente a partir da variação da posição do pico de difração, dada pela equação 2 ,

$\epsilon \quad \frac{d \quad d}{d} \cong-\Delta \theta \quad \operatorname{cotg} \theta_{0}$ sendo $\theta$ a posição do pico de difração correspondente à distância interplanar $d$ do material sob tensão e $\theta_{0}$ a posição do pico de difração correspondente à distância interplanar $d_{0}$ do material livre de tensões. O tensor completo das tensões residuais pode ser obtido através da medida da deformação do material nas três direções principais, conforme equação 3 e Figura 4 [11],

$\sigma_{i}=\frac{E_{h k l}\left(1-v_{h k l}\right)}{\left(1+v_{h k l}\right)\left(1-2 v_{h k l}\right)} \varepsilon_{i}+\frac{v_{h k} E_{h k l}}{\left(1-2 v_{h k l}\right)\left(1+v_{h k l}\right)} \sum_{j} \varepsilon_{j}, j=1,2,3$ and $j \neq i$

sendo $\sigma_{i}, \varepsilon_{i}$ as tensões e deformações nas direções principais, $E_{h k l} e v_{h k l}$ o módulo de Young e o coeficiente de Poisson na direção cristalográfica perpendicular aos planos de difração $\{\mathrm{hkl}\}$. Como se pode ver na Figura 4, consegue-se medir nas diferentes direções alterando a direção da amostra em relação aos feixes incidente e difratado. Para garantir que o material irradiado seja sempre o mesmo para as diferentes orientações é muito importante que as medidas sejam realizadas com ângulos de difração perto dos $90^{\circ}$. Tipicamente podem ser obtidas distribuições de tensões residuais por difração de neutrões em amostras de alumínio com espessuras até cerca de $150 \mathrm{~mm}$ e em amostras de aço com espessuras até cerca de $35 \mathrm{~mm}$.

\section{STRESS-SPEC: Instrumento para Análise de Tensões}

Os resultados experimentais obtidos por difração de neutrões apresentados neste trabalho foram realizados no instrumento

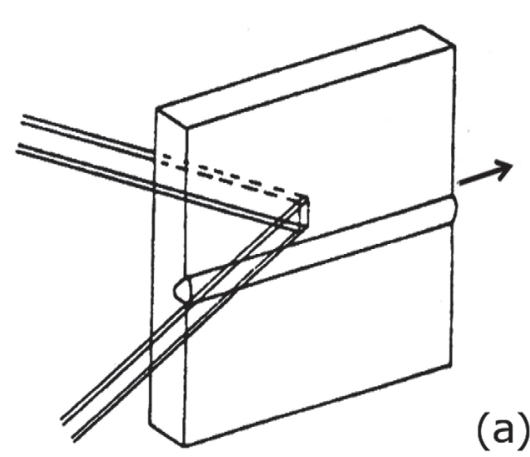

(a)

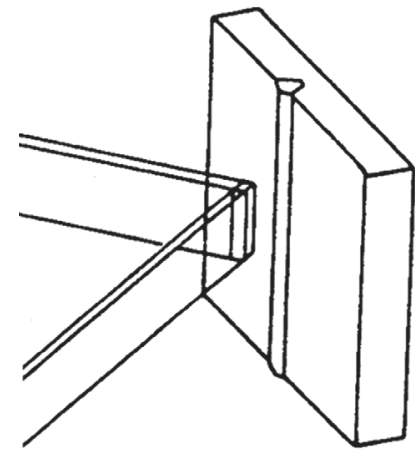

(b)

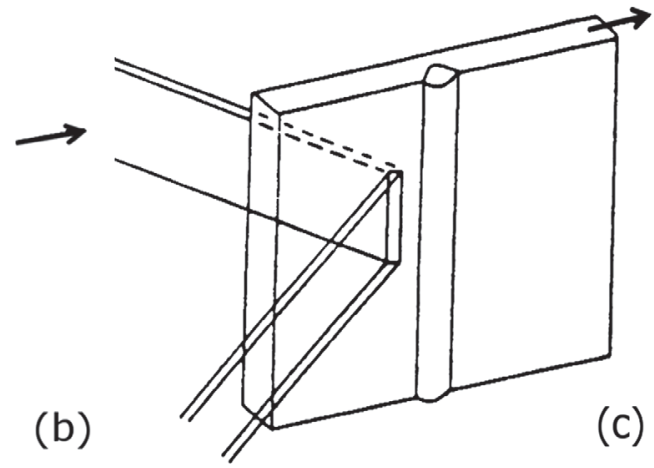

(c)

Figura 4. Direções de medida com feixe de neutrões em relação à direção da soldadura: (a) longitudinal, (b) normal e (c) transversal. 
STRESS0SPEC (Figura 5) existente no reator nuclear FRM II da Universidade Técnica de Munique. Este instrumento foi expressamente desenvolvido e projetado para o estudo da textura e das tensões residuais $[4,12]$. O instrumento está equipado com três monocromadores (grafite, germânio e silício), os quais permitem variar o comprimento de onda entre $0,1 \mathrm{~nm}$ e $0,24 \mathrm{~nm}$, de modo a permitir obter ângulos de difração de $90^{\circ}$, tipicamente usados na determinação de tensões com feixe de neutrões para os principais materiais em aplicações de engenharia. O elemento de volume analisado é definido, neste instrumento, por um sistema de fendas e colimadores nos feixes incidente e difratado, podendo a fenda no feixe incidente tomar valores desde $1 \times 1 \mathrm{~mm}^{2}$ até $5 \times 20 \mathrm{~mm}^{2}$ e a largura da fenda no feixe difratado variar continuamente até $15 \mathrm{~mm}$.

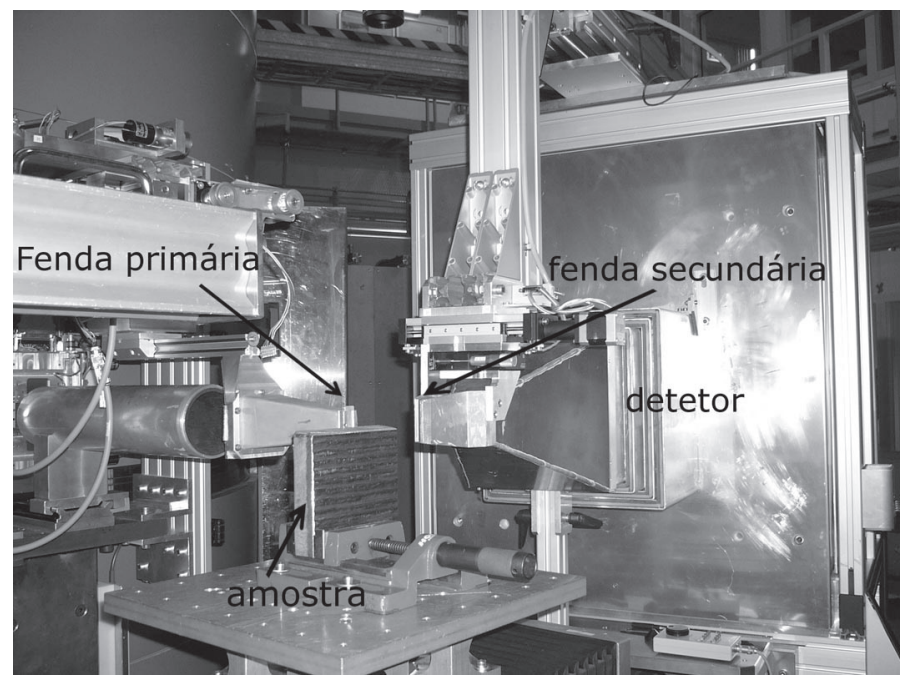

Figura 5. Instrumento STRESS-SPEC, FRM II -TUM.

A mesa onde são colocadas as amostras para análise é motorizada, permitindo movimentos com precisão de $10 \mu \mathrm{m}$ e suportando amostras até $300 \mathrm{~kg}$. O equipamento está ainda equipado com um detetor de neutrões de localização linear o que permite reduzir o tempo de análise.

Para o estudo das texturas cristalográficas, o instrumento está equipado com um robot STÄUBLI que suporta peças até $30 \mathrm{~kg}$ e que permite rodar a peça em todas as direcções sem constrangimentos de colisões com as fendas do difratómetro [12]. O instrumento está também equipado com uma máquina de tração/compressão de $50 \mathrm{kN}$, desenvolvida especificamente para o STRESS-SPEC nas oficinas do FRM II, que permite o estudo das propriedades mecânicas dos materiais durante ensaios de tração e de compressão em simultâneo com a difração de neutrões.

\section{Materiais e Condições Experimentais}

Em determinadas aplicações industriais, tal como é o caso da indústria química, os equipamentos de processo por estarem em contacto com fluidos corrosivos necessitam de proteção das suas paredes interiores contra a corrosão. Uma das soluções normalmente adotada na conceção e fabrico dos equipamentos de processo é proporcionada pela deposição de camadas de soldadura de aços inoxidáveis, resistentes aos agentes agressivos, sobre aços C-Mn ferríticos, menos onerosos, mas com resistência mecânica adequada $[13,14]$. Os principais problemas decorrentes da deposição destas camadas são os fenômenos de fissuração na interface aço inoxidável/aço carbono, que afetam a integridade e segurança dos equipamentos [15]. O mecanismo de fissuração é geralmente associado à migração de carbono que ocorre na interface entre o aço inoxidável e o aço base ferrítico, sendo agravado na presença de tensões residuais de tração. A migração de carbono para o aço inoxidável inicia-se durante as operações de soldadura e continua durante o tratamento térmico realizado após soldadura, dando oportunidade para a formação de microestruturas complexas, principalmente martensite e carbonetos. Por outro lado, em virtude das diferenças da condutividade térmica e dos coeficientes de expansão térmica dos materiais de base e do material depositado, resultam tensões residuais, quer devido ao próprio processo de produção do revestimento, quer devido às diferenças de ciclos térmicos induzidas nas diferentes regiões $[16,17]$. Habitualmente, após a produção dos revestimentos por soldadura, são realizados tratamentos térmicos, tendo em vista a redução do nível de tensões induzidas nas amostras revestidas.

Neste trabalho, as amostras foram preparadas a partir de placas quadradas em aço EN 100283P355 NH com $300 \mathrm{~mm}$ de lado e $20 \mathrm{~mm}$ de espessura. Uma das superfícies foi revestida por soldadura de arco submerso com elétrodos de $4 \mathrm{~mm}$ de diâmetro em aço austenítico, tendo sido feitas três camadas de soldadura sobrepostas. Na Tabela 1 estão descritas as composições químicas do material base e dos elétrodos usados na elaboração das amostras. Na primeira camada foi usado um elétrodo EN 12072S 23122 L (aço austenítico 309 L) para minimizar problemas metalúrgicos de diluição do material base (aço ferrítico) e a camada de soldadura que se pretendia depositar (aço austenítico $316 \mathrm{~L}$ ). Na segunda e terceira camadas foi usado um elétrodo EN 12072 - S19123 L. Com este procedimento as amostras ficaram com uma espessura final de $27,5 \mathrm{~mm}$. A composição do fluxo e os procedimentos de soldadura e estão descritos na Tabela 2.

Após soldadura uma das amostras foi submetida a um tratamento térmico de relaxação de tensões, a uma temperatura de $620^{\circ} \mathrm{C}$ durante 1 hora, tendo-se feito o arrefecimento no forno, até à temperatura ambiente. Esta amostra recebeu a referência TT620, enquanto a amostra não tratada termicamente foi referenciada por STT. Posteriormente ao tratamento térmico, as placas foram cortadas por eletroerosão em 4 provetes com dimensões $150 \times 150 \mathrm{~mm}^{2}$ destinados à caraterização metalúrgica, à caraterização das tensões residuais por difração de neutrões, por difração de raios $\mathrm{X}$ e pelo método do furo incremental, e ao estudo da resistência à corrosão. Neste artigo serão apenas apresentados os resultados de tensões obtidos por difração de neutrões.

Os perfis de tensões residuais foram determinados no instrumento STRESS-SPEC com recurso a um monocromador de Si (400), cuja curvatura se pode alterar horizontalmente para aumentar a resolução conforme o ângulo de difração a usar. As aquisições foram determinadas com um feixe monocromático com comprimento de onda de $0,166 \mathrm{~nm}$. A análise de tensões 
Tabela 1. Composição dos materiais usados

\begin{tabular}{|c|c|c|c|c|c|c|c|c|c|c|}
\hline $\begin{array}{c}\text { Material } \\
{[\% \text { em massa }]}\end{array}$ & $\mathrm{C}$ & $\mathrm{Si}$ & $\mathrm{Mn}$ & $\mathrm{P}$ & $\mathrm{S}$ & $\mathrm{Cr}$ & $\mathrm{Ni}$ & $\mathrm{Mo}$ & $\mathrm{Cu}$ & $\mathrm{N}$ \\
\hline $\begin{array}{c}\text { EN 10028-3 } \\
\text { P355 NH }\end{array}$ & 0,18 & 0,33 & 1,12 & 0,015 & 0,002 & 0,04 & 0,16 & - & 0,21 & 0,004 \\
\hline $\begin{array}{c}\text { EN 12072 } \\
\text { S 23 12 2 L }\end{array}$ & 0,008 & 0,29 & 1,4 & 0,016 & 0,003 & 21,3 & 14,8 & 2,57 & 0,11 & 0,057 \\
\hline $\begin{array}{c}\text { EN 12072 } \\
\text { S 19 12 2 L }\end{array}$ & 0,018 & 0,38 & 1,8 & 0,016 & 0,009 & 18 & 11,7 & 2,54 & 0,07 & 0,055 \\
\hline
\end{tabular}

Tabela 2. Parâmetros usados no processo de soldadura de arco submerso

\begin{tabular}{|c|c|c|c|c|}
\hline \multicolumn{2}{|c|}{ Pré-aquecimento } & \multicolumn{3}{|c|}{ Por chama $\left(150^{\circ} \mathrm{C}\right)$} \\
\hline \multicolumn{2}{|c|}{ Intensidade Corrente } & \multicolumn{3}{|c|}{$300 \mathrm{~A}$} \\
\hline \multicolumn{2}{|c|}{ Voltagem } & \multicolumn{3}{|c|}{$29-30 \mathrm{~V}$} \\
\hline \multicolumn{5}{|c|}{ Composição do fluxo [\%] } \\
\hline $\mathrm{SiO}_{2}$ & $\mathrm{MgO}$ & $\mathrm{CaF}_{2}$ & $\mathrm{Al}_{2} \mathrm{O}_{3}$ & $\mathrm{Cr}$ \\
\hline 31.0 & 25.0 & 6.0 & 13.0 & 4.8 \\
\hline
\end{tabular}
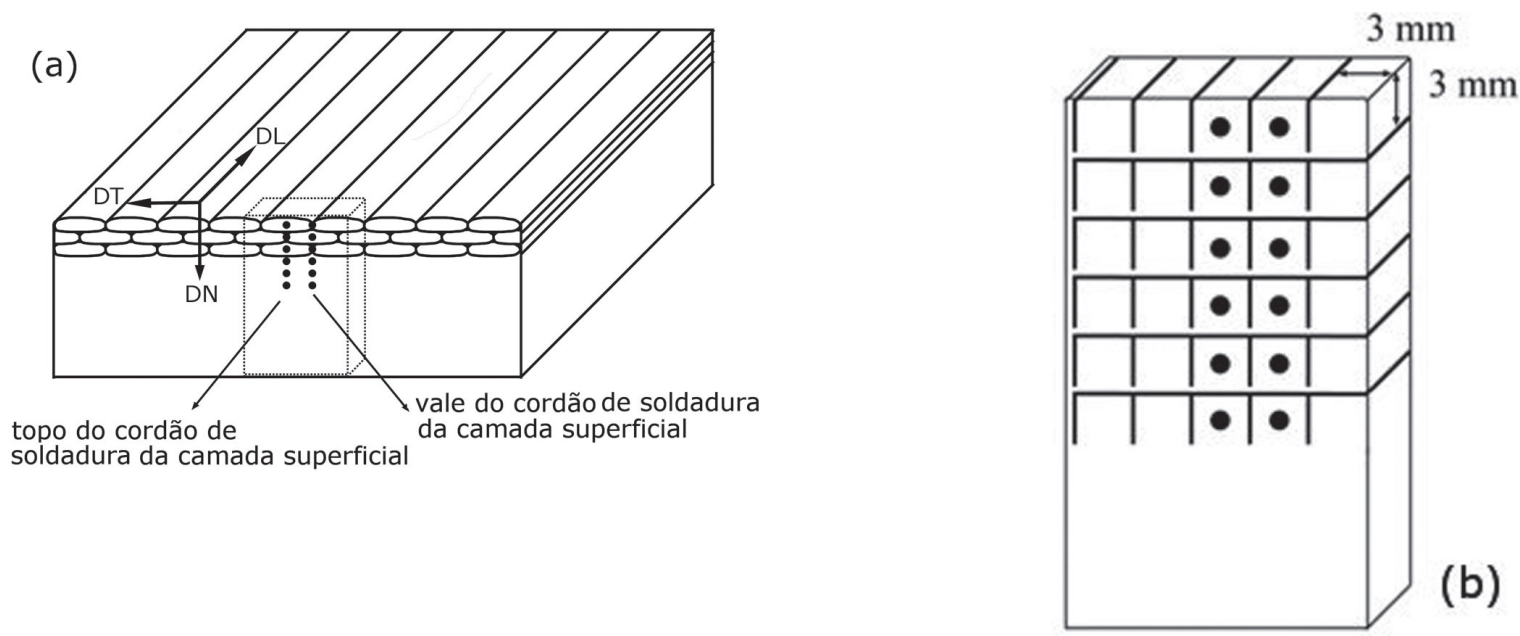

Figura 6. (a) Definição das direções de medida: DL, DT e DN, correspondentes às direções longitudinal, transversal e normal à direção da soldadura; (b) Esquema do "pente" com os pequenos cubos para a determinação da distância interplanar do material sem tensões, $d_{0}$.

residuais foi efetuada usando o pico de difração $\{211\}$ da fase ferrítica $\left(2 \theta \approx 91.4^{\circ}\right)$ e o pico de difração $\{311\}$ da fase austenítica $\left(2 \theta \approx 101^{\circ}\right)$. Em cada medida na direção longitudinal ao cordão de soldadura caraterizou-se um volume de material com largura de $2 \mathrm{~mm}, 2 \times 2 \times 2 \mathrm{~mm}^{3}$, usando uma fenda de $2 \times 2 \mathrm{~mm}^{2}$ no feixe primário e uma fenda com largura de $2 \mathrm{~mm}$ no feixe secundário. As medidas nas direções transversal e normal foram realizadas num volume de material irradiado de $2 \times 10 \times 2 \mathrm{~mm}^{3}$, sendo, neste caso, utilizada uma fenda de $2 \times 10 \mathrm{~mm}^{2}$ no feixe primário e uma fenda com largura de $2 \mathrm{~mm}$ no feixe secundário. Para minimizar a influência da operação de corte por nas tensões residuais determinadas nas amostras, procurou-se que as aquisições fossem realizadas em pontos afastados das arestas do corte.

Para caracterizar as amostras por difração de neutrões, fo- ram determinadas as deformações nas direções longitudinal, transversal e normal à soldadura, tendo sido efetuadas aquisições na vertical do topo e do vale da última camada depositada, conforme se esquematiza na Figura 6 (a). As tensões residuais foram determinadas usando a Eq. 3, considerando as seguintes constantes elásticas $\mathrm{E}_{211}=220 \mathrm{GPa}, v_{211}=0.28$ e $\mathrm{E}_{311}=175 \mathrm{GPa}$, $v_{311}=0.31$ para a direção perpendicular aos planos cristalinos $\{211\}$ do pico Fe $\alpha$ e $\{311\}$ do Fe- $\gamma$, respetivamente [18]. Note-se que a difração é uma técnica seletiva, e por isso as constantes radiocristalográficas dependem dos planos cristalinos $\{\mathrm{hkl}\}$. Para a determinação do parâmetro $d_{0}$ das amostras, que carateriza o material sem tensões, foram retirados de cada uma das amostras, por eletroerosão, pequenos cubos com $3 \mathrm{~mm}$ de aresta, representativos do material existente nas diferentes profundida- 
des examinadas, conforme se mostra na Figura 6 (b). Com este procedimento pretende-se e admite-se que os cubos fiquem sem tensões, sendo considerados como referência para a obtenção do valor do $d_{0}$ para cada profundidade de medida. Apresenta-se na Figura 7 o "pente" com os pequenos cubos para a determinação do valor de $d_{0}$, retirado da amostra tratada termicamente. Optou-se por não cortar completamente os cubos, deixando-os parcialmente ligados ao "pente", pois desta forma mantém-se a referência exata da profundidade que está a ser analisada.

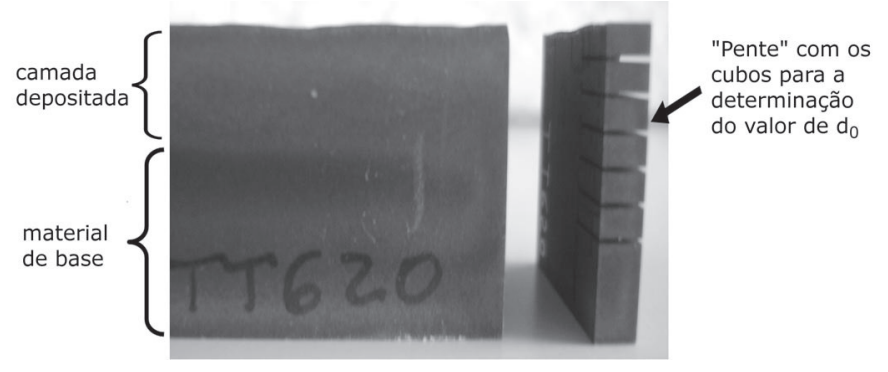

Figura 7. Amostra para a determinação da distância interplanar, $d_{0}$, do material sem tensões

\section{Resultados Experimentais}

As figuras 8 a 11 apresentam a evolução, ao longo da espessura das amostras, das tensões residuais obtidas por difração de neutrões nas direções longitudinal. Os resultados apresentados correspondem aos valores determinados em cada uma das fases, austenítica (correspondente ao revestimento), e ferrítica (correspondente ao material base), pelo que aparecem nas imagens com simbologia diferente. Para obter o estado de tensão global do material, estes resultados terão de ser combinados, tomando em consideração a fração volúmica de cada uma das fases existente a cada uma das profundidades analisadas, frações essas que podem ser quantificadas por difração de raios X, por exemplo.

Os perfis de tensões residuais determinados na amostra sem tratamento térmico, ao longo da vertical do topo e do vale de um cordão de soldadura da camada superficial, são apresentados na Figura 8 e na Figura 9, na direção longitudinal e transversal, respetivamente. Observou-se a presença de tensões de tração em ambas as regiões do material depositado, até uma profundidade na ordem de $7 \mathrm{~mm}$, passando depois a tensões de compressão. No material tensões residuais longitudinais são pouco intensas na vizinhança da interface, sendo nulas em pontos mais afastados. Na zona do topo da soldadura as tensões atingem os 200 a $250 \mathrm{MPa}$, tanto em tração como em compressão, o que corresponde aproximadamente ao limite de elasticidade do aço AISI 316. Já as tensões residuais na zona do vale apresentam valores máximos em tração na ordem de $500 \mathrm{MPa}$, que correspondem a valores na ordem da resistência à tração do aço AISI 316, o que só pode ser explicado por uma deformação plástica local significativa ou por sobrestimação do método de avaliação. A técnica de difração de neutrões proporcionou uma evolução suave do perfil de tensões, com pequena dispersão. A Figura 8 mostra que na zona de interface entre a austenite e a ferrite há alguma discrepância entre as tensões obtidas de um e outro lado da interface, a qual pode ser atribuída em parte ao microestruturas de estruturas aí presentes. Embora já existam algumas publicações sobre a determinação de tensões residuais em soldaduras com recurso à técnica de difração de neutrões [19, 20], a utilização desta técnica em revestimentos por soldadura está ainda pouco documentada. Contudo, encontrou-se um artigo recente [21], sobre um aço inoxidável austenítico revestido por soldadura com aço ferrítico, o que representa a situação inversa da aqui estudada, onde se observou que as tensões residuais medidas na interface apresentavam discrepâncias similares.

Os resultados obtidos após o tratamento térmico são apresentados na Figura 10 e Figura 11, também para uma zona de topo e de vale do revestimento, na direção longitudinal e transversal, respetivamente. Observou-se que as tensões de tração na camada austenítica foram parcialmente relaxadas, passando as tensões de tração a tensões de compressão na camada mais próxima da superfície. Este efeito é particularmente favorável no caso de estruturas sujeitas à fadiga [22], ou à corrosão sob tensão [23]. Quando são comparados os valores de tensões residuais obtidos a maiores profundidades na amostra TT620 com os valores da amostra sem tratamento térmico, verifica-se apenas uma ligeira redução das tensões residuais de tração. Na região mais próxima da interface, do lado do aço inoxidável, observou-se uma pequena variação do nível de tensões residuais, permanecendo estas contudo em compressão. Do lado do material ferrítico, próximo da interface, observou-se que estas passaram a ser de compressão, o que tem por efeito reduzir a tendência à fissuração nesta zona. As tensões residuais medidas na espessura apresentam também neste caso uma evolução suave, sendo a dispersão mais elevada nas medições feitas na fase austenítica, na zona do vale. A interpretação destes resultados carece de uma melhor quantificação da fração volúmica das duas fases aí presentes, e de uma melhor avaliação do valor de $\mathrm{d}_{0}$, trabalho atualmente em curso no CEMDRX, através de metodologias que utilizam a difração de raios $X$.

Saliente-se que a difração de neutrões possibilita a determinação do perfil de tensões residuais através da espessura da amostra de uma forma não destrutiva, contrariamente à difração de raios X que implica a remoção de sucessivas camadas do material quando se pretende obter o perfil das tensões em profundidade. Outra vantagem da difração de neutrões é a rapidez na aquisição dos dados experimentais. Contudo as experiências com feixe de neutrões são extraordinariamente onerosas e por isso o acesso a tempo de feixe nos reatores é muito condicionado. Normalmente o acesso a estes grandes centros de investigação, conhecidos por "large-scale facilities", faz-se através de candidaturas de acesso, em datas pré-estabelecidas, as quais são avaliadas por um comité de peritos internacionais que decidem a sua aprovação em função da fundamentação e qualidade das propostas de candidatura apresentadas.

Os resultados apresentados nesta publicação fazem parte de um estudo mais vasto no qual, para além da difração de neutrões, se recorre às técnicas de determinação de tensões residuais por difração de raios X e pelo método do furo incremental [24]. As três técnicas são complementares e permitirão uma melhor compreensão da influência dos parâmetros dos tratamentos térmicos, após o processo de soldadura, no campo de tensões residuais das amostras, não depreciando contudo os resultados apresentados neste estudo. 

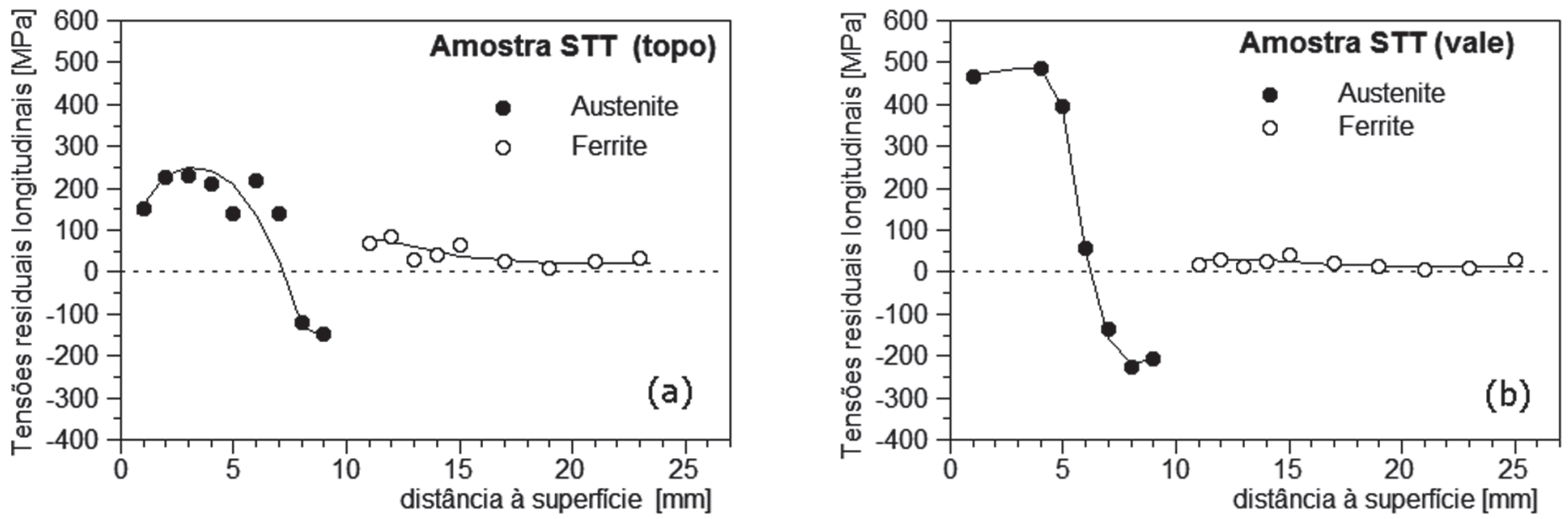

Figura 8. Tensões residuais segundo a direção longitudinal do cordão de soldadura na amostra STT: (a) distribuição ao longo da vertical passando pelo topo dum cordão de soldadura da última camada depositada e (b) distribuição ao longo da vertical passando por um vale do cordão de soldadura da última camada depositada.
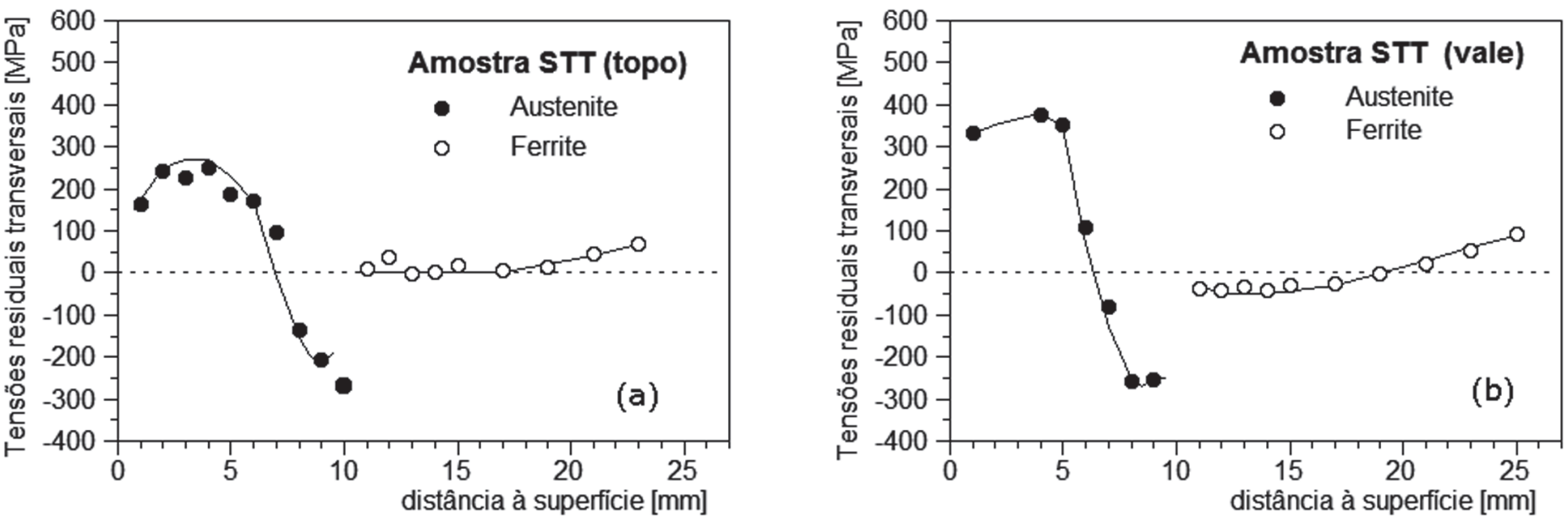

Figura 9. Tensões residuais segundo a direção transversal do cordão de soldadura na amostra STT: (a) distribuição ao longo da vertical passando pelo topo dum cordão de soldadura da última camada depositada e (b) distribuição ao longo da vertical passando por um vale do cordão de soldadura da última camada depositada.
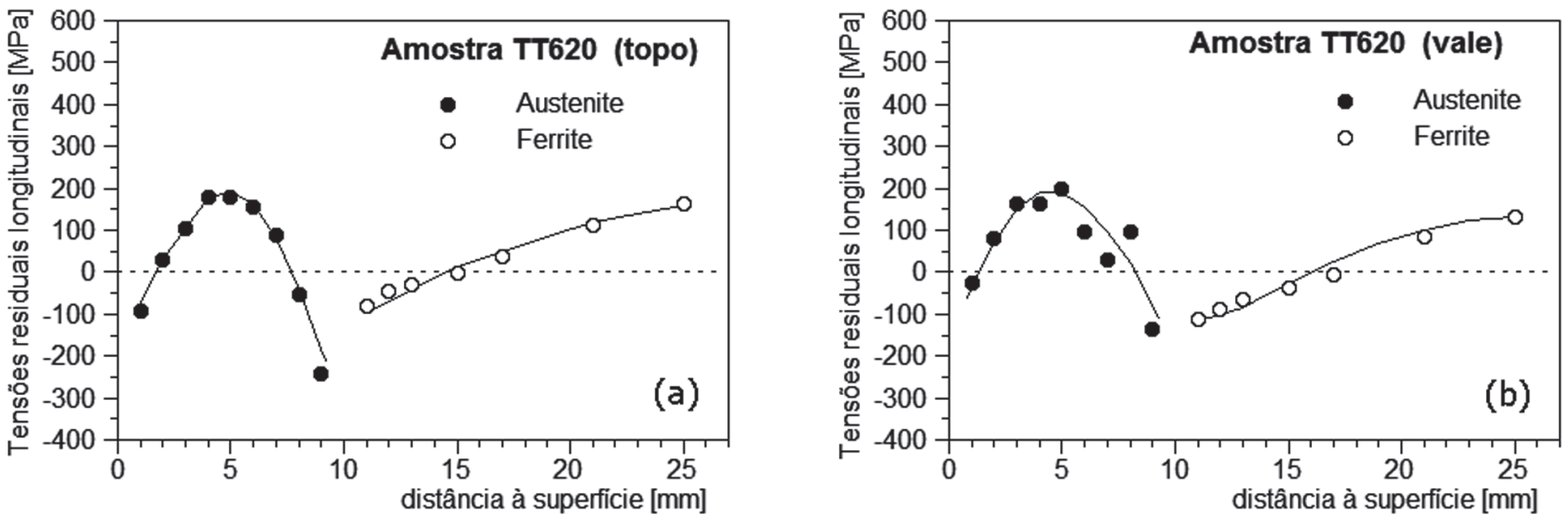

Figura 10. Tensões residuais segundo a direção longitudinal do cordão de soldadura na amostra TT620: (a) distribuição ao longo da vertical passando pelo topo dum cordão de soldadura da última camada depositada e (b) distribuição ao longo da vertical passando por um vale do cordão de soldadura da última camada depositada. 

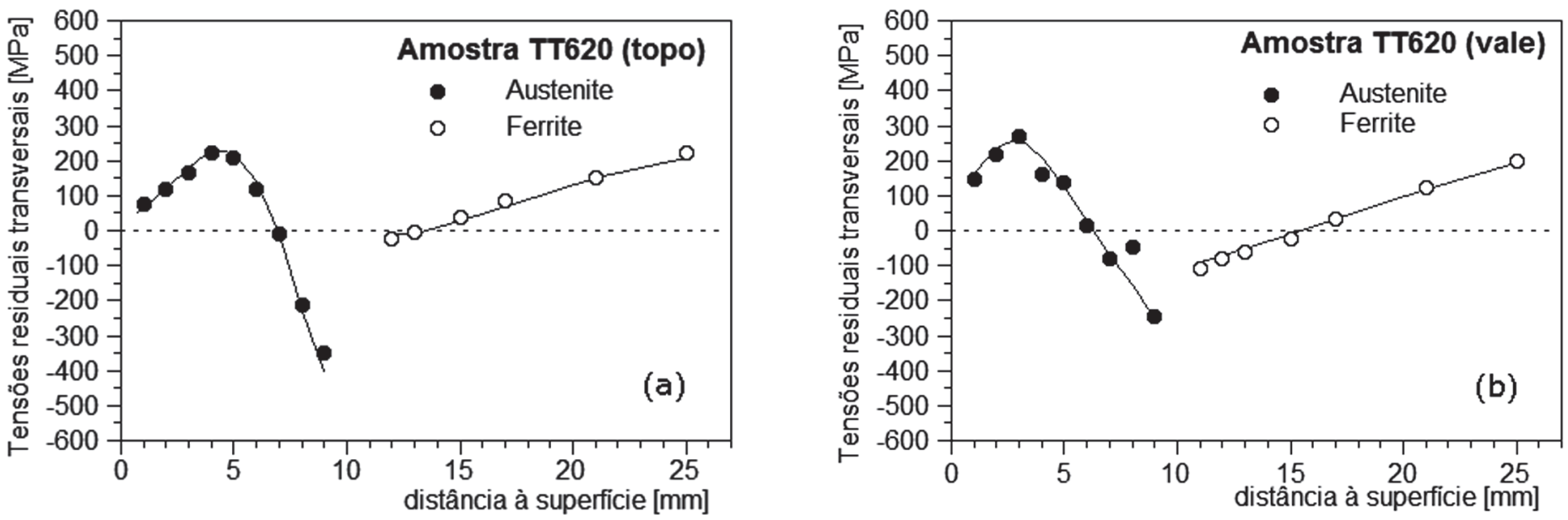

Figura 11. Tensões residuais segundo a direção transversal do cordão de soldadura na amostra TT620: (a) distribuição ao longo da vertical passando pelo topo dum cordão de soldadura da última camada depositada e (b) distribuição ao longo da vertical passando por um vale dos do cordão soldadura da última camada depositada.

\section{Conclusões}

Neste trabalho foi apresentada a técnica da difração de neutrões para a análise de tensões residuais em revestimentos produzidos por soldadura. Esta técnica é especialmente vocacionada para o estudo das tensões residuais induzidas por soldadura, pois permite obter a distribuição de tensões em profundidade sem necessidade de destruir a amostra.

Como exemplo de aplicação da técnica, selecionou-se o estudo da influência dum tratamento térmico realizado em amostras de aço ferrítico revestidas por soldadura de arco submerso com aços austeníticos. Os resultados obtidos indicam que o tratamento térmico das amostras à temperatura de $620^{\circ} \mathrm{C}$ induziu uma relaxação de tensões nas regiões analisadas e a técnica permitiu quantificar essa relaxação. Os resultados das tensões residuais na região da interface do revestimento com o material de base parecem também indicar uma diminuição das tensões residuais de tração na fase ferrítica. Contudo os resultados nesta região necessitam de ser avaliados tendo em conta a fração volúmica das fases presentes e uma melhor avaliação do valor da distância interplanar do material sem tensões $\left(\mathrm{d}_{0}\right)$, trabalhos esses que estão atualmente em curso, recorrendo a técnicas de difração de raios $\mathrm{X}$.

\section{Agradecimentos}

Os autores agradecem ao Forschungs-Neutronenquelle Heinz Maier-Leibnitz (FRM II), Garching, Alemanha, a concessão de tempo de feixe e à Comissão Europeia o financiamento da missão através do $6^{\circ}$ Programa Quadro, "Strengthening the European Research Area, Research Infrastructures", Contract RII3-CT-2003-505925.

Os autores agradecem o apoio financeiro do Fundo Europeu de Desenvolvimento Regional (FEDER) através do "Programa Operacional Fatores de Competitividade - COMPETE" e fundos nacionais da "Fundação para a Ciência e a Tecnologia (FCT)", projetos PTDC/EME-TME/103845/2008 e PestC/FIS/
UI0036/2011.

À empresa ARSOPI, Vale de Cambra, Portugal, os autores agradecem o fornecimento das amostras estudadas.

\section{Referências}

[1] LU, J., Handbook of measurement of residual stresses, Society for Experimental Mechanics, Inc., The Fairmont Press, Inc., Lilburn, Georgia, USA, 1996.

[2] HUTCHINGS, M.T. et al., Introduction to the Characterization of Residual Stress by Neutron Diffraction, CRC Press, 2005.

[3] FITZPATRICK, M. E. and LODINI, A. Analysis of Residual Stress by diffraction using Neutron and Synchrotron Radiation, Taylor \& Francis, 2003.

[4] HOFMANN, M. et al., The New Materials Science Diffraktometer STRESS-SPEC at FRM II, Mater. Sci. Forum, Vol. 524525, p. 211-216, 2006.

[5] http://www.helmholtz-berlin.de/forschung/funkma/mikro/ methoden/neutronendiffraktometer/index_de.html,

acesso em 9 Janeiro 2013.

[6] STUHR U. et al., Time-of-flight diffraction with multiple frame overlap Part II: The strain scanner POLDI at PSI, Nuclear Instruments and Methods in Physics Research A, 545, p. 330338, 2005.

[7] SANTISTEBAN J.R. et al., ENGIN X: a third-generation neutron strain scanner, J. Applied Crystallography 39, p. 812825, 2006.

[8] CERRETI M., Recent development for strain measurements at LLB, Physica B, 276-278, 2000, p. 932933.

[9] PIRLING T., BRUNO G. and WITHERS P.J., SALSA, a new concept for strain mapping at the ILL", Mat. Sci. Eng. A, 437, p. 139-144, 2006.

[10] ANDERSON I.S., MCGREEW R. and BILHEUX H.Z., Neutron Imaging and Applications, a reference for the imaging community, (EDS), Chapter 2 Neutron sources and facilities, Springer Science+Business Media LLC, 2009. 
[11] VIKTOR, H., Structural and Residual Stress Analysis by Nondestructive Methods: Evaluation, Application, Assessment, Elsevier Science B. V., Amsterdam, 1997.

[12] BROKMEIER, H.-G. et al., Texture analysis at neutron diffractometer STRESS-SPEC, Nuclear Instruments \& Methods in Physics Research, Section A: Accelerators, Spectrometers, Detectors, and Associated Equipment, Vol. 642, p. 87-92, 2011.

[13] KUMAR, R. et al., Qualification of stainless steel cladding for nuclear application, Transactions of the Indian institute of metals, 56 (1), p. 61-67, 2011.

[14] MURAGAN, N. and PARMAR, R. S. Stainless steel cladding deposited by automatic gas metal arc welding, Welding Journal, 76 (10), S391-S403, 1997.

[15] HOHE, J.; BRAND, M. and SIEGELE, D., Investigation on residual stresses and fracture behaviour of cladded plates with defects, Proc. of IIW Int. Conf. Safety and Reliability of Welded Components in Energy and Processing Industry, Graz - Austria, p. 303-308, 2008.

[16] VEIGA, C.; LOUREIRO, A. and DIAS, A. Residual stress evolution in repair welds, International Journal for Strain Measurement, 39, p. 57-63, 2003.

[17] YEN, H. J.; LIN, M. C. C. and CHEN, L. J., Measurement and simulation of residual stress in type 304 weld overlay stainless steel pipe, Int. J. Pressure Vessels and Piping, 60 (3), p. 271283, 1994.

[18] EIGENMANN, B. ; MACHERAUCH, E., Mat.-wiss. u. Werkstofftech. 27, p. 426, 1996.

[19] WOO, W. et al., Residual stress determination in a thick ferritic steel weld plate using neutron diffraction, J Mater Sci, 47 (14), p. 5617-5623, 2012.

[20] HAIGH, R.D. et al., Neutron diffraction residual stress measurements on girth-welded 304 stainless steel pipes with weld metal deposited up to half and full pipe wall thickness, International Journal of Pressure Vessels and Piping, 101, p. 1-11, 2013.

[21] TARAN, Yu V, et al., Residual stresses in a shape welded steel tube by neutron diffraction, J. Phys.: Condens. Matter 20 (10), 104258, 2008.

[22] SINDHU R.A. et al., Effects of residual stresses on the static and fatigue strength of laser-welded lap joints with different welding speeds, International Journal of Automotive Technology, Vol. 11, (6), p. 857-863, 2010.

[23] LU J.Z., et al., Effects of laser peening on stress corrosion cracking (SCC) of ANSI 304 austenitic stainless steel, Corrosion Science, Vol. 60, p. 145-152, 2012.

[24] MARQUES, M. J. et al., Residual Stresses Profiles of Cladded Austenitic Stainless Steel evaluated by Xray Diffraction and by Incremental Hole Drilling Method, 9th International Conference on Residual Stresses, ICRS9, Garmisch-Partenkirchen, Alemanha, 7 a 9 de outubro de 2012, aceite para publicação, Materials Science Forum, 2013. 